\title{
Impaired local regulation of ryanodine receptor type 2 by protein phosphatase 1 promotes atrial fibrillation
}

\author{
David Y. Chiang ${ }^{1,2,3 \dagger}$, Na Li ${ }^{1,2 \dagger}$, Qiongling Wang ${ }^{1,2}$, Katherina M. Alsina ${ }^{1,2}$, Ann P. Quick ${ }^{1,2}$, \\ Julia O. Reynolds ${ }^{1,2}$, Guoliang Wang ${ }^{1}$, Darlene Skapura ${ }^{1,2}$, Niels Voigt ${ }^{4}$, Dobromir Dobrev ${ }^{4}$, \\ and $X$ ander H.T. Wehrens ${ }^{1,2,5 *}$
}

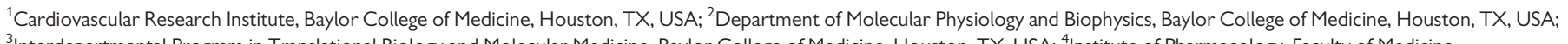
${ }^{3}$ Interdepartmental Program in Translational Biology and Molecular Medicine, Baylor College of Medicine, Houston, TX, USA; ${ }^{4}$ Institute of Pharmacology, Faculty of Medicine, University Duisburg-Essen, Essen, Germany; and ${ }^{5}$ Department of Medicine (Cardiology), Baylor College of Medicine, Houston, TX, USA

Received 4 March 2014; revised 7 April 2014; accepted 25 April 2014; online publish-ahead-of-print 8 May 2014

Time for primary review: 7 days

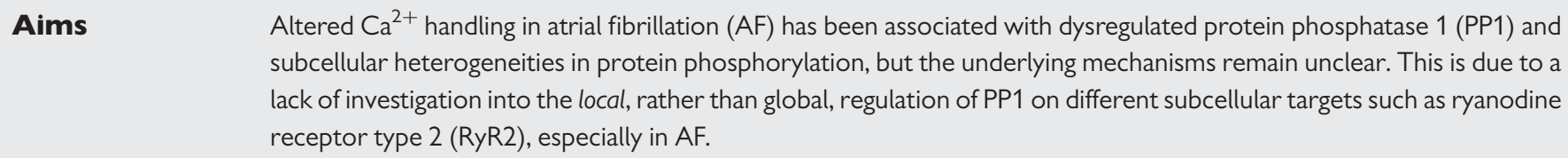

Methods We tested the hypothesis that impaired local regulation of PP1 causes RyR2 hyperphosphorylation thereby promoting and results $\quad A F$ susceptibility. To specifically disrupt PP1's local regulation of RyR2, we used the spinophilin knockout (Sp ${ }^{-1-}$ ) mice (Mus musculus) since PP1 is targeted to RyR2 via spinophilin. Without spinophilin, the interaction between PP1 and RyR2 was reduced by $64 \%$, while RyR2 phosphorylation was increased by $43 \%$ at serine (S) 2814 but unchanged at S2808. Lipid bilayer experiments revealed that single RyR2 channels isolated from Sp ${ }^{-1-}$ hearts had an increased open probability. Likewise, $\mathrm{Ca}^{2+}$ spark frequency normalized to sarcoplasmic reticulum $\mathrm{Ca}^{2+}$ content was also enhanced in $\mathrm{Sp}^{-1}$ atrial myocytes, but normalized by $\mathrm{Ca}^{2+}$ /calmodulin-dependent protein kinase II (CaMKII) inhibitors KN-93 and AIP and also by genetic inhibition of RyR2 S2814 phosphorylation. Finally, $\mathrm{Sp}^{-1-}$ mice exhibited increased atrial ectopy and susceptibility to pacing-induced AF, both of which were also prevented by the RyR2 S2814A mutation.

\section{Conclusion} PP1 regulates RyR2 locally by counteracting CaMKII phosphorylation of RyR2. Decreased local PP1 regulation of RyR2 contributes to RyR2 hyperactivity and promotes AF susceptibility. This represents a novel mechanism for subcellular modulation of calcium channels and may represent a potential drug target of AF.

Keywords Atrial fibrillation • Protein phosphatase 1 - Ryanodine receptor type 2 • Spinophilin $\bullet \mathrm{Ca}^{2+} /$ calmodulindependent kinase II

\section{Introduction}

Atrial fibrillation (AF) is the most common sustained arrhythmia, associated with an increased risk of stroke and all-cause mortality. Previously, studies have found that protein phosphatase 1 (PP1) is dysregulated in $\mathrm{AF}$ patients, and that this is associated with inhomogeneous protein phosphorylation levels across different subcellular domains. ${ }^{1}$ The PP1 holoenzyme is the main serine/threonine PP in the heart and consists of a highly conserved catalytic subunit (PP1c) and a regulatory subunit, of which there are close to 200 known. $^{2}$ A number of previous studies have tried to elucidate the role of PP1 in cardiac pathophysiology by either overexpressing PP1 $\mathrm{c}^{3}$ or altering its inhibitors such as protein inhibitors 1 (I-1) and 2 (I-2). ${ }^{4,5}$ While these studies have generated much interest and uncovered potential therapeutic strategies, they were conducted in the context of cardiac hypertrophy and heart failure, and not AF. Moreover, these studies did not directly address 
the observation that the PP levels are different across subcellular compartments in AF patients. ${ }^{1}$ For example, while the total activity of PP1 in chronic AF patients is elevated, ${ }^{1}$ the phosphorylation level of ryanodine receptor type 2 (RyR2) is also paradoxically increased. ${ }^{6,7}$ These observations underscore an incomplete understanding of PP1's function at the subcellular level, and suggest that PP1 locally regulates $\mathrm{Ca}^{2+}$ handling proteins within specific microdomains.

Recent studies have shown that a hyperphosphorylated and hyperactive RyR 2 is one of the main drivers of AF pathogenesis, by contributing to inappropriate diastolic sarcoplasmic reticulum (SR) $\mathrm{Ca}^{2+}$ release and triggered activity. ${ }^{6-9}$ Hyperphosphorylation of RyR2 has generally been attributed to hyperactivity of RyR2-bound kinases, such as protein kinase A (PKA) and $\mathrm{Ca}^{2+}$ /calmodulin-dependent protein kinase II (CaMKII). ${ }^{6,7,9}$ However, the steady-state RyR2 phosphorylation reflects a delicate balance between kinases and PPs, namely PP1 and PP2A, which are also found in the RyR2 macromolecular complex. ${ }^{10,11}$ While several studies have documented the global protein and/or activity levels of PP1 in patients with cardiac diseases including AF, ${ }^{1,4,12,13}$ little is known about the mechanisms by which PP1 regulates RyR2 locally, especially in the context of AF.

Since the target specificity of PP1 depends on its regulatory subunit and spinophilin is the regulatory subunit that targets PP1c to RyR2, ${ }^{14}$ we used spinophilin knockout $\left(\mathrm{Sp}^{-1-}\right)$ mice to study how PP1 locally regulate RyR2. ${ }^{11}$ We tested the hypothesis that, in the absence of spinophilin, decreased PP1-mediated dephosphorylation of RyR2 leads to RyR2 hyperactivity, thereby contributing to AF pathogenesis. Our data showed that reduced PP1 regulation of RyR2 enhanced phosphorylation of serine (S)2814 but not S2808 on RyR2, which was also associated with increased RyR2 activity. Furthermore, this was associated with increased ectopic activity and susceptibility to pacing-induced AF in mice, both of which were rescued with genetic ablation of the S2814 phosphorylation site on RyR2. Finally, we further validated our findings in vitro by showing that overexpression of spinophilin leads to selective dephosphorylation of RyR2 at S2814 but not at S2808. Taken together, these findings demonstrate for the first time the importance of PP1's local regulation of RyR2, as it relates to AF susceptibility.

\section{Methods}

Further details are provided in Supplementary material online.

\subsection{Study animals}

All animal studies were performed according to the protocol (AN-4044) approved by the Institutional Animal Care and Use Committee of the Baylor College of Medicine conforming to the Guide for the Care and Use of Laboratory Animals. All animals used in this study were Mus musculus maintained on a C57BL/6 background for more than 10 generations. Spinophilin knockout mice $\left(\mathrm{Sp}^{-1-}\right)$ and RyR2 S2814A (S2814A) knockin mice were generated previously. ${ }^{6,14} \mathrm{We}$ intercrossed $\mathrm{Sp}^{-1-}$ mice with $\mathrm{S} 2814 \mathrm{~A}$ mice to obtain the double-mutant $\mathrm{Sp}^{-1-}:$ S2814A mice. Wild-type (WT), $\mathrm{Sp}^{-1-}$, and $\mathrm{Sp}^{-1-}: \mathrm{S} 2814 \mathrm{~A}$ mice between the ages of 2 and 4 months and from both sexes were used for the experiments. Mice were anaesthetized using $1.5-2 \%$ isoflurane in $95 \% \mathrm{O}_{2}$ during telemeter implantation, intracardiac electrophysiology studies, echocardiography studies, and prior to cervical dislocation for tissue harvesting.

\subsection{Western blotting}

Protein extraction and western blotting were performed as previously described. ${ }^{15}$ Details are provided in Supplementary material online.

\subsection{Co-immunoprecipitation}

Cardiac SR preparation and immunoprecipitation were performed based on previously described methods. ${ }^{16}$ Details are provided in Supplementary material online.

\subsection{Single-channel recordings}

Single-channel recordings were obtained under voltage-clamp conditions at $0 \mathrm{mV}$ as previously described. ${ }^{17}$ Details are provided in Supplementary material online.

\section{5 $\mathrm{Ca}^{2+}$ imaging}

Single atrial myocytes were isolated using a modified collagenase method as described. ${ }^{18}$ Details are provided in Supplementary material online.

\subsection{Telemetry ECG recordings}

To record ECG from conscious unrestrained mice aged 2-4 months, telemeters (Data Sciences International, MN, USA) were implanted intraperitoneally as previously described. ${ }^{19}$ The recordings were analysed using the ECG-Auto software (Emka Technologies) and spontaneous atrial ectopic events were counted between 12 a.m. and 5 a.m.

\subsection{Intracardiac electrophysiology in mice}

In vivo electrophysiology studies were performed in mice at the age of 2-4 months as previously described. ${ }^{20} \mathrm{AF}$ inducibility was determined by using an overdrive pacing protocol and defined as the occurrence of rapid and fragmented atrial electrograms with irregular RR intervals for at least $1 \mathrm{~s}$.

\subsection{Molecular cloning and cell culture}

Spinophilin was cloned from a cardiac cDNA library and co-transfected with RyR2 (gift from Dr Andrew Marks, Columbia University), and CaMKII (gift from Dr Mark Anderson, University of lowa) into HEK293 cells maintained in DMEM medium (Invitrogen, Carlabad, CA, USA) supplemented with 10\% foetal bovine serum, $1 \%$ penicillium and streptomycin, and $2 \mathrm{mM}$ L-glutamine. Details are provided in Supplementary material online.

\subsection{Statistical analysis}

Data are presented as mean \pm SEM. One-way ANOVA followed by the post hoc Bonferroni test and category Fisher's exact test were applied, where appropriate; otherwise, two-tailed Student's t-test was applied. A $P$-value $\leq 0.05$ was considered statistically significant.

\section{Results}

\subsection{Decreased association of PP1 with RyR2 in spinophilin-deficient mice}

To confirm the existence of spinophilin in cardiac tissue, we first evaluated the endogenous expression of spinophilin in the mouse heart, in particular the mouse atria. Using western blotting, we detected spinophilin expression in both the atria and ventricles of WT mice, and found that its protein level was significantly higher in the atria (see Supplementary material online, Figure S1). In order to selectively interrupt the interaction between RyR2 and PP1 without altering the global levels of PP1, we used $\mathrm{Sp}^{-1-}$ mice for our studies. ${ }^{14}$ Western blotting confirmed that the global protein levels of RyR2, PP1, and PP2A were unchanged in $\mathrm{Sp}^{-1-}$ atria (Figure 1A). Furthermore, the levels of other key $\mathrm{Ca}^{2+}$-handling proteins were also unaltered (Figure 1B).

The initial report that PP1 is targeted to RyR2 via spinophilin was based on artificial in vitro assays where GST-RyR2 fusion proteins were overexpressed and used for co-immunoprecipitation. ${ }^{11}$ Since then (2001), no study has repeated the findings nor demonstrated this interaction in vivo. To establish that the interaction between RyR2 and PP1 is 

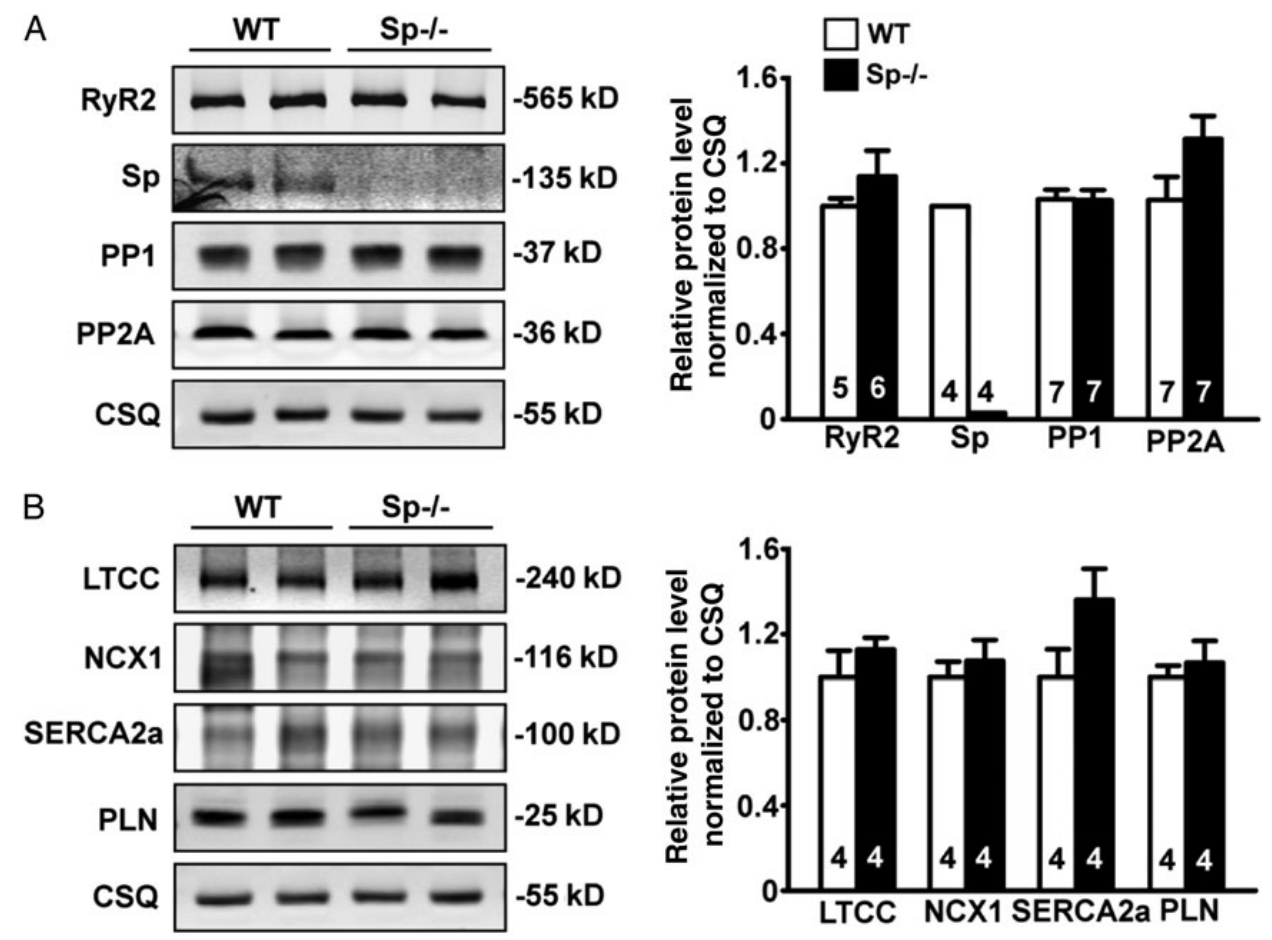

C
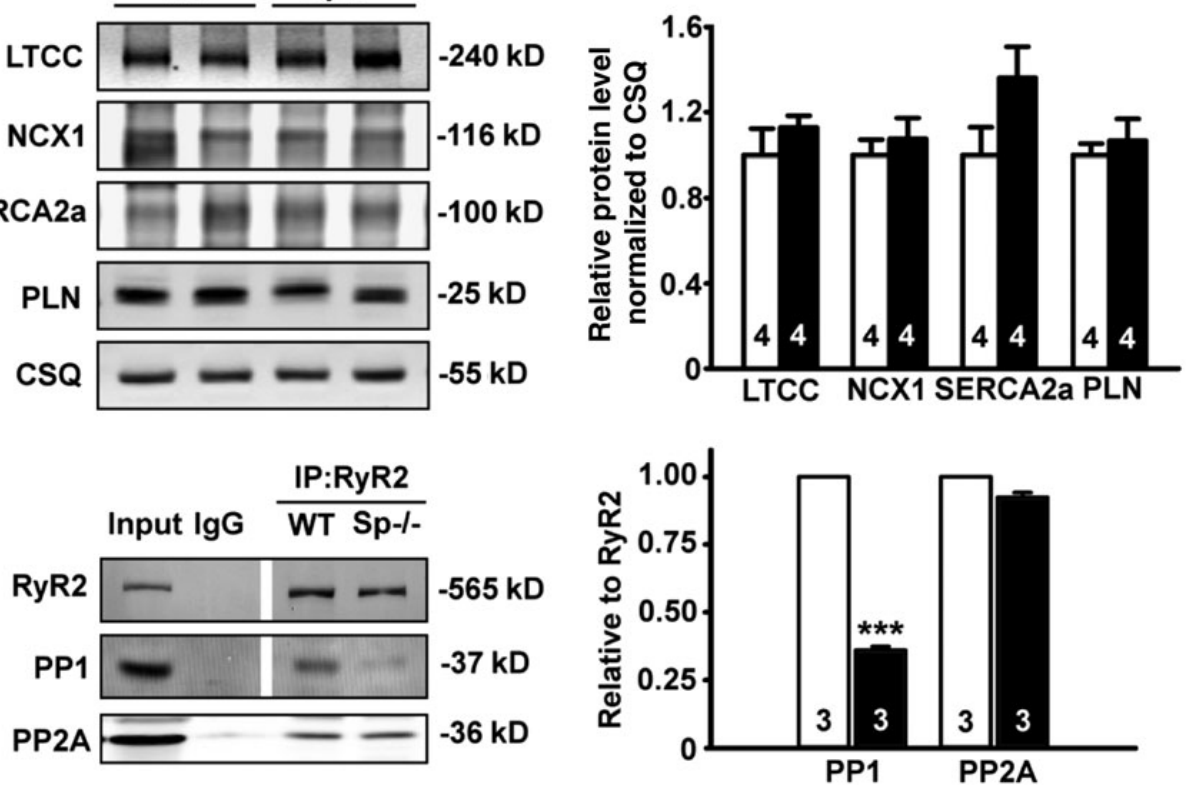

Figure I Decreased association of PP1 with RyR2 in the absence of spinophilin. Representative western blots and quantification showing that with spinophilin ablation. (A) The protein levels of RyR2, PP1, and PP2A are unaltered. (B) The protein levels of other key $\mathrm{Ca}^{2+}{ }_{-h a n d l i n g}$ proteins are also unchanged and $(C)$ the association between RyR2 and PP1, but not between RyR2 and PP2A, is significantly decreased. $* * * P<0.001$ vs. WT.

mediated by spinophilin in vivo, we performed co-immunoprecipitation (IP) and showed that the amount of PP1 associated with RyR2 was reduced by $64 \%(P<0.001)$ in the absence of spinophilin (Figure 1C). However, even with extensive washing as evident by the absence of signal in the lgG control lane, there was residual PP1 that was pulled-down with RyR2. In contrast, the amount of PP2A that was co-immunoprecipitated with RyR2 was unaltered (Figure 1C), suggesting that spinophilin deficiency specifically altered RyR2 regulation by PP1 and not PP2A.

To further study the disruption of this interaction between RyR2 and PP1 in the absence of spinophilin in the native cellular environment, we performed immunocytochemistry in isolated atrial myocytes from $\mathrm{Sp}^{-1-}$ and WT mice with antibodies against RyR2 and PP1 (see Supplementary material online, Figure S2A). We found a subtle but noticeable difference in the PP1 signal between the $\mathrm{SP}^{-1-}$ and $\mathrm{WT}$ atrial myocytes, where the PP1 signal in green was more scattered and less regularly formed in a striated pattern (see Supplementary material online, Figure $S 2 B$ ). This observation was confirmed by quantifying the co-localization between RyR2 and PP1 using the correlation $R$ coefficient, which is significantly lower in the $\mathrm{Sp}^{-1-}$ cells $(0.40 \pm 0.02)$ vs. WT cells $(0.56 \pm$ $0.02 ; P<0.001$; see Supplementary material online, Figure S2C).

\subsection{Impaired PP1 local regulation enhances RyR2 phosphorylation at S2814}

Since the amount of PP1 bound to RyR2 was decreased in $\mathrm{Sp}^{-1-}$ hearts (Figure 1C), we initially anticipated that RyR2 at $\mathrm{S} 2808$ would be increased based on a previous report. ${ }^{21}$ However, western blotting using phospho-specific antibodies revealed that RyR2 phosphorylation at S2808 was not significantly altered in $\mathrm{Sp}^{-1-}$ mice (Figure $2 \mathrm{~A}$ ). In contrast, phosphorylation of the primary CaMKII site $\$ 2814$ was elevated by $43 \%(P<0.05)$. In order to test whether changes in phosphorylation were specific for RyR2 in the absence of spinophilin, we also measured the phosphorylation status of phospholamban (PLN), which is also in the cardiac SR. Consistent with the model that spinophilin targets PP1 to RyR2 and not to PLN, we did not find any significant changes in the phosphorylation of PLN at both S16 and T17 in the absence of spinophilin (see Supplementary material online, Figure S3). This further supports the notion that spinophilin deficiency specifically impairs PP1's local regulation of RyR2.

To further assess whether the hyperphosphorylation of RyR2 at S2814 was caused by changes in the kinases, we measured both the cytosolic protein and activity levels of PKA and CaMKII in WT and $\mathrm{Sp}^{-1-}$ mice 

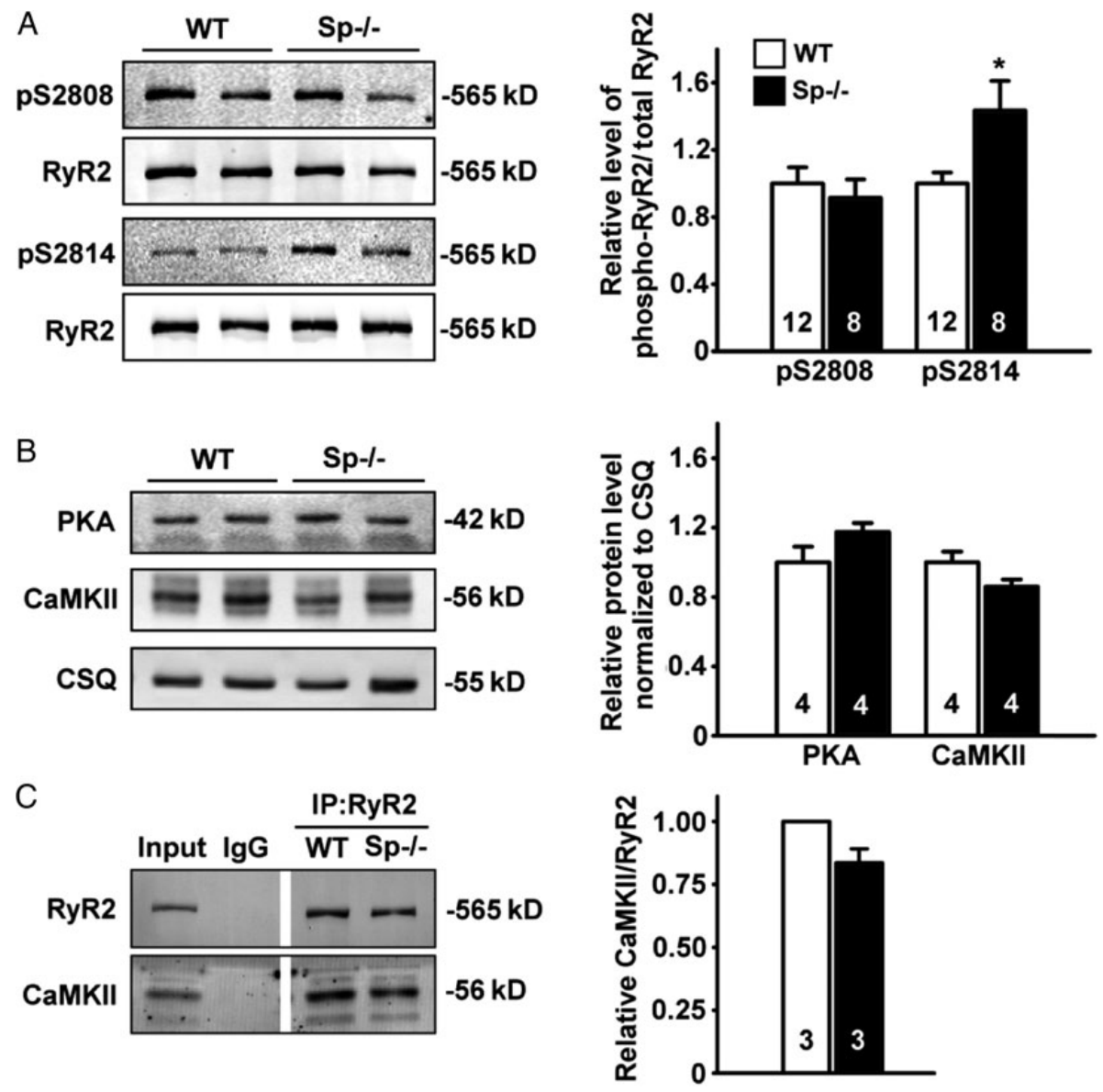

Figure 2 Impaired local regulation by PP1 leads to increased phosphorylation of RyR2 at S2814. Representative western blots and quantification showing (A) increased phosphorylation of RyR2 at S2814 (pS2814) but not at S2808 (pS2808) in atria from Sp ${ }^{-1-}$ mouse, (B) unchanged global protein levels of PKA and $\mathrm{CaMKII}$, and $(C)$ unaltered level of $\mathrm{CaMKII}$ in the RyR2 complex as determined by co-immunoprecipitation. $* P<0.05$ vs. WT.

and found no significant changes (Figure 2B and see Supplementary material online, Figure S4). Furthermore, since the CaMKII site S2814 was hyperphosphorylated (Figure 2A), we performed co-immunoprecipitation and found no increase in the amount of CaMKII binding to RyR2 (Figure 2C). Taken together, these data suggest that PP1 primarily regulates S2814 on RyR2 in vivo and that disruption of PP1 binding to RyR2 promotes hyperphosphorylation of RyR2 at S2814.

\subsection{Impaired PP1 local regulation enhances RyR2 channel activity}

Since changes in RyR2 phosphorylation status can alter channel activity, ${ }^{6}$ we assessed the activity of single RyR2 channels from the $\mathrm{Sp}^{-1-}$ and WT hearts in planar lipid bilayers. The open probability (Po) of RyR2 channels from $\mathrm{Sp}^{-1-}$ mice was significantly higher $(\mathrm{Po}=0.10 \pm 0.02)$ compared with WT $(P o=0.01 \pm 0.01 ; P<0.01$; Figure $3 A$ and $B$ ). The mean open time was also higher for RyR2 channels from $\mathrm{Sp}^{-1-}$ mice (18.6 \pm $7.6 \mathrm{~ms})$, compared with RyR2 from WT mice $(1.2 \pm 0.3 \mathrm{~ms} ; P<0.05)$. Finally, there was a non-significant trend towards a reduced mean closed time for RyR2 from Sp ${ }^{-1-}$ mice (246 $\left.\pm 82 \mathrm{~ms}\right)$ compared with channels from WT mice ( $3818 \pm 3333 \mathrm{~ms} ; P=\mathrm{NS}$; Figure 3 C and D).

To assess RyR2 activity in the cellular environment, we performed $\mathrm{Ca}^{2+}$ spark experiments in atrial myocytes isolated from $\mathrm{Sp}^{-1-}$ and WT mice. After loading the myocytes with Fluo-4-AM, a Ca ${ }^{2+}$-sensitive dye, we measured the $\mathrm{Ca}^{2+}$ spark frequency (CaSF) as well as determined the SR $\mathrm{Ca}^{2+}$ load using a caffeine dump protocol. ${ }^{22}$ At baseline following $1-\mathrm{Hz}$ pacing, there was a significant increase in CaSF normalized to SR Ca ${ }^{2+}$ load in $\mathrm{Sp}^{-1-}$ atrial myocytes (1.7 \pm 0.2 a.u.) compared with WT $(0.8 \pm 0.2$ a.u.; $P<0.05$; Figure $4 A$ and $B)$. When CaMKII was activated by pacing the myocytes at a higher frequency of $3 \mathrm{~Hz},{ }^{6}$ there was an even greater increase in CaSF in $\mathrm{Sp}^{-1-}$ myocytes $(3.5 \pm$ 0.6 a.u.) compared with WT $(1.7 \pm 0.2$ a.u.; $P<0.05$; Figure $4 C$ ). Taken together, these results suggest that, with impaired local PP1 regulation, enhanced RyR2 activity promotes spontaneous SR $\mathrm{Ca}^{2+}$ release in isolated cardiomyocytes.

\subsection{Mechanistic basis of RyR2 hyperactivity with impaired PP1 regulation}

To gain more insights into the mechanisms underlying enhanced RyR2 phosphorylation with impaired PP1 regulation, we applied CaMKII inhibitors to $\mathrm{Sp}^{-1-}$ myocytes (Figure 4). Two different CaMKII inhibitors (KN-93 and AIP) both reduced the higher CaSF levels in myocytes from $\mathrm{Sp}^{-1-}$ mice to levels similar to those observed in WT myocytes $(0.9 \pm 0.2$ a.u. for $\mathrm{KN}-93$ and $0.9 \pm 0.3$ a.u. for AIP; $P<0.05$ vs. $\mathrm{SP}^{-1-}$ for both; Figure $4 A$ and $B$ ). In contrast, the inactive analogue of $\mathrm{KN}-93, \mathrm{KN}-92$, did not reduce the CaSF in myocytes from $\mathrm{Sp}^{-1-}$ mice $\left(2.1 \pm 0.3\right.$ a.u., $P=N S$ vs. $\mathrm{Sp}^{-1-}$; Figure $\left.4 \mathrm{~B}\right)$. 
A WT

Open

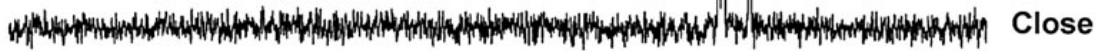

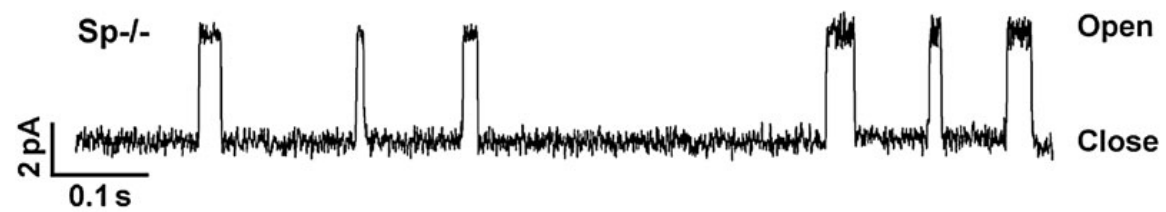

B

WT $\square \mathrm{Sp}-\mathrm{I}-$

C

D
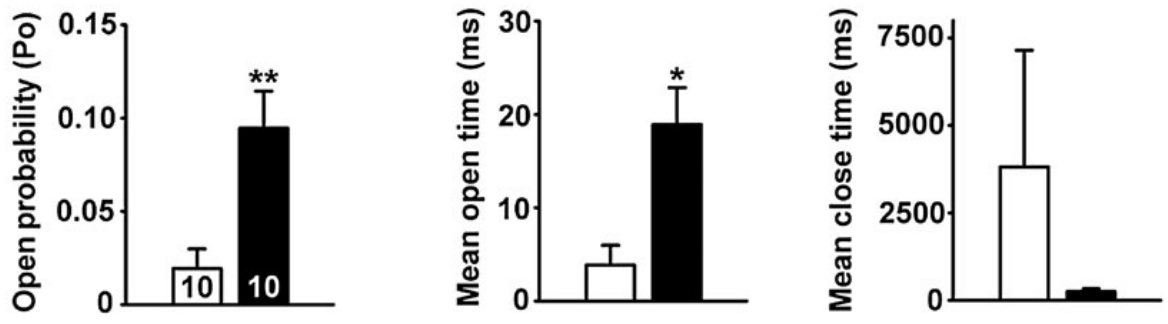

Figure 3 Increased RyR2 open probability with impaired local regulation by PP1. (A) Representative single-channel recordings of RyR2 from WT and $\mathrm{Sp}^{-1-}$ mouse hearts. $(B)$ Increased open probability, $(C)$ increased mean open time, and $(D)$ decreased mean close time of single RyR2 channels from $\mathrm{Sp}^{-1-}$ hearts. Numbers within the bars indicate the number of channels studied from three animals per group. $* P<0.05$, $* * P<0.01$ vs. WT.

A

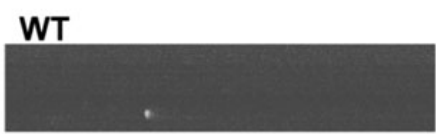

Sp-I-

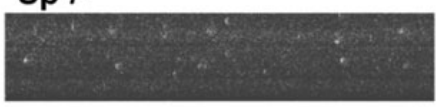

Sp-I- + KN-93

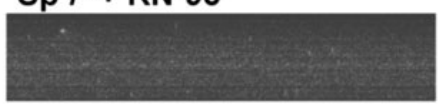

Sp-I- + KN-92

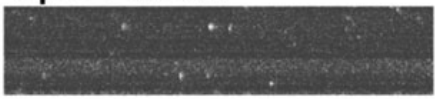

Sp-I- + AIP

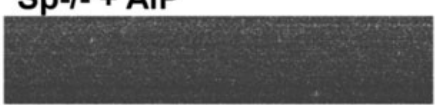

Sp-/-:S2814A

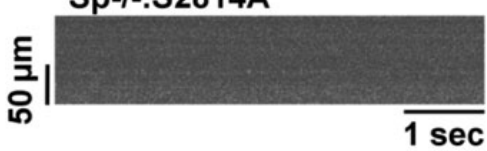

B

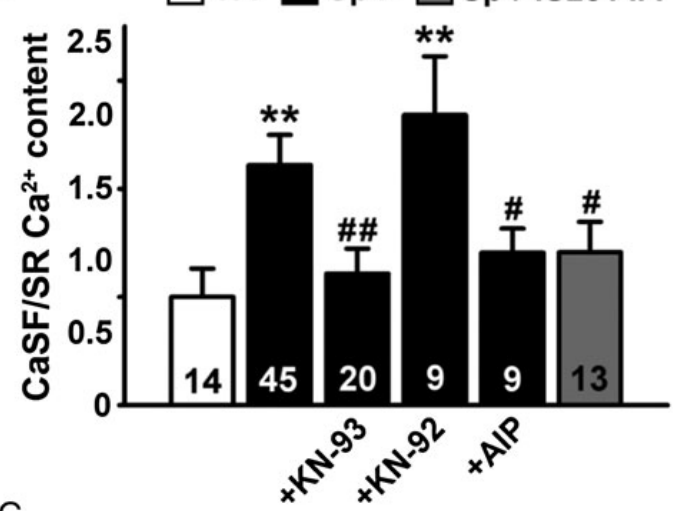

C

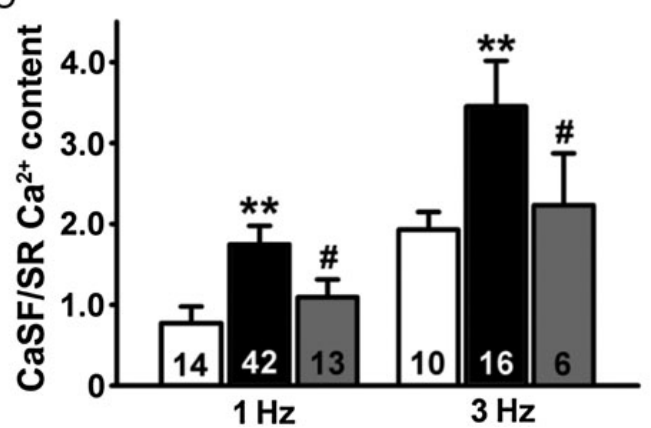

Figure 4 Impaired local regulation by PP1 increased CaSF in atrial myocytes. (A) Representative confocal line-scan images of atrial myocytes loaded with Fluo4-AM. (B) Bar graph summarizing the ratio of CaSF normalized to SR load for atrial myocytes isolated from WT and Sp ${ }^{-1-}$ mice, in the presence or absence of CaMKII inhibitors KN-93 or AIP, or control drug KN-92. (C) Bar graph comparing the ratio of CaSF to SR load in atrial myocytes paced at $1 \mathrm{~Hz}$ (same data as from panel $B$ ) or at $3 \mathrm{~Hz}$. Numbers in the bars indicate the number of cells studied from 2 to 12 mice. ${ }^{* *} P<0.01 \mathrm{vs}$. WT. ${ }^{\#} P<0.05$, ${ }^{\# \#} \mathrm{P}<0.01$ vs. Sp ${ }^{-1-}$. 


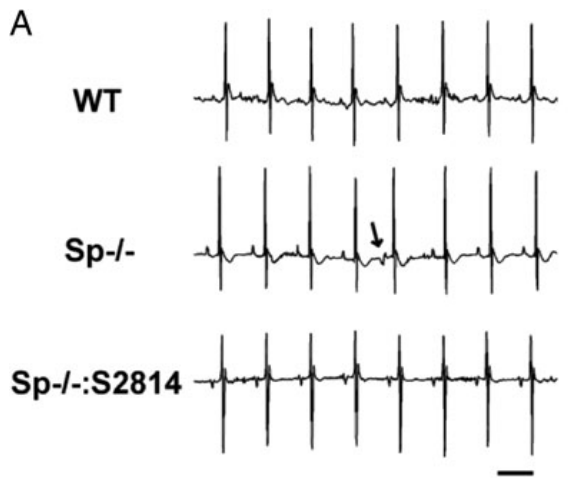

B $\square$ wT $\square$ Sp-I- $\square$ sp-I:S2814

$100 \mathrm{~ms}$

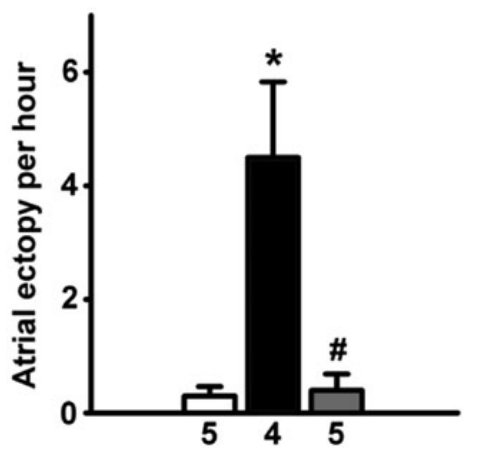

Figure 5 Impaired regulation of RyR2 by PP1 increased atrial ectopy in mice. (A) Representative telemetry ECG recordings of conscious, freely roaming mice. Arrow points to an atrial ectopic beat in a Sp ${ }^{-1-}$ mouse. (B) Bar graph summarizing the number of atrial ectopic beats per hour in WT, $\mathrm{Sp}^{-1-}$, and $\mathrm{Sp}^{-1-}$ :S2814A mice. Numbers under the bars indicate the number of mice studied. $* P<0.05$ vs. WT. ${ }^{\#} p<0.05$ vs. Sp ${ }^{-1-}$

Since phosphorylation of the $\mathrm{S} 2814$ site was increased in $\mathrm{Sp}^{-1-}$ mice (Figure 2A), we sought to assess whether genetic inactivation of the S2814 phosphorylation in $\mathrm{Sp}^{-1-}$ mice could attenuate the increased RyR2 activity. Therefore, $\mathrm{Sp}^{-1-}$ mice were crossed with mice in which the S2814 phosphorylation site on RyR2 was inactivated by mutation to alanine (S2814A). $\mathrm{Ca}^{2+}$ spark measurements in atrial myocytes from $\mathrm{Sp}^{-1-}$ :S2814A mice revealed significantly reduced CaSF levels $(1.1 \pm 0.2$ a.u.) compared with those in myocytes from $S \mathrm{P}^{-1-}$ mice $(1.7 \pm 0.2$ a.u.; $P<0.05$; Figure $4 A$ and $B)$. The same rescue effect was also observed when the atrial myocytes were paced at the higher frequency of $3 \mathrm{~Hz}\left(2.2 \pm 0.6\right.$ vs. $3.5 \pm 0.6$ a.u. for $\mathrm{Sp}^{-1-}$; $P<0.05$; Figure 4C). Taken together, these data demonstrated that abnormal RyR2-mediated SR $\mathrm{Ca}^{2+}$ release events observed in $\mathrm{Sp}^{-1-}$ atrial myocytes could be normalized by CaMKII inhibition or genetic ablation of S2814 phosphorylation on RyR2. These data support the concept that PP1 regulates RyR2 primarily at the S2814 site and that this directly counteracts CaMKII phosphorylation of RyR2.

\subsection{Impaired PP1 regulation predisposes mice to atrial ectopy and AF induction}

Previous studies have associated RyR2 hyperphosphorylation and hyperactivity with $A F$ in various animal models. ${ }^{6,8,18,23}$ Since our data demonstrate that RyR2 is both hyperphosphorylated (Figure 2) and hyperactive with impaired PP1 regulation (Figures 3 and 4), we assessed whether $\mathrm{Sp}^{-1-}$ mice are more prone to developing AF. ECG telemetry revealed that $\mathrm{Sp}^{-1-}$ mice exhibited a larger number of ectopic beats $(4.5 \pm 1.3$ events $/ h)$ compared with WT mice $(0.3 \pm 0.2$ events $/ h$; $P<0.05$; Figure 5). In contrast, there was a reduced number of ectopic beats in $\mathrm{Sp}^{-1-}:$ S2814A mice $\left(0.4 \pm 0.3\right.$ events/h, $P<0.05$ vs. Sp ${ }^{-1-}$; Figure 5). Baseline ECG parameters are provided in Supplementary material online, Table S1.

To determine whether $\mathrm{Sp}^{-1-}$ mice were also more susceptible to develop $A F$, we subjected them to programmed electrical stimulation ${ }^{18}$ and found that $A F$ was induced in $83 \%$ of the $\mathrm{Sp}^{-1-}$ mice compared with only $25 \%$ of the WT mice $(P<0.05$; Figure 6$)$. In contrast, the $\mathrm{Sp}^{-1-}: \mathrm{S} 2814 \mathrm{~A}$ mice were resistant to pacing-induced AF (14.3\% vs. $\mathrm{Sp}^{-1-}$; Figure 6). Taken together, these findings suggest that $\mathrm{Sp}^{-1-}$ mice are more susceptible to AF as a result of abnormal RyR2 phosphorylation due to reduced PP1-mediated dephosphorylation of S2814.

\subsection{Absence of structural remodelling in atria of $\mathrm{Sp}^{-1-}$ mice}

Additional experiments were performed to determine whether the enhanced susceptibility to $\mathrm{AF}$ in $\mathrm{Sp}^{-1-}$ mice could be attributed to structural remodelling of the atria. Histological analysis was performed on hearts from WT and $\mathrm{Sp}^{-1-}$ mice obtained at the age of 2-4 months. Haematoxylin-eosin stained longitudinal heart sections revealed the absence of gross morphological changes in $\mathrm{Sp}^{-1-}$ hearts, although they were slightly smaller overall, consistent with their smaller body size as previously reported (see Supplementary material online, Figure S5A). ${ }^{14}$ Echocardiography revealed normal cardiac ejection fractions, and slightly reduced end-systolic and end-diastolic diameters (ESD and EDD) of $\mathrm{Sp}^{-1-}$ hearts (both $P<0.01$ vs. WT; see Supplementary material online, Table S2). However, when normalized to the total body weight, the heart weight-to-body weight ratio (HW/BW) was actually slightly increased in $\mathrm{Sp}^{-1-}$ mice (see Supplementary material online, Figure S5B). This was mainly due to a minor but statistically significant increase in the ventricular weight-to-body weight ratio (VW/BW; $P<0.05$ vs. WT; see Supplementary material online, Figure S5C). Importantly, however, the atrial weight-to-body weight ratio (AW/BW) was unaltered in $\mathrm{Sp}^{-1-}$ mice compared with WT mice $(P=\mathrm{NS}$; see Supplementary material online, Figure S5D). Finally, Masson's trichome staining revealed no difference in the amount of atrial fibrosis, comparing atria from $\mathrm{Sp}^{-1-}$ and WT mice (see Supplementary material online, Figure S6). Taken together, these findings suggest that $\mathrm{Sp}^{-1-}$ mice are more susceptible to AF as a result of abnormal RyR2 phosphorylation due to reduced PP1-mediated dephosphorylation of S2814.

\subsection{In vitro validation of PP1-spinophilin targeting of RyR2 S2814}

To further validate our findings which suggest that PP1-spinophilin targets RyR2 specifically at S2814 and not at S2808 (Figure 2A), we co-transfected HEK293 cells with constructs containing RyR2 and CaMKII with either spinophilin or an empty vector. Using the western blot, we first confirmed the overexpression of spinophilin, while levels of CaMKII and PP1 were not significantly changed (Figure 7A). Next, we measured the phosphorylation level of RyR2 at S2808 and S2814 and found a significant decrease only at S2814 in the presence of 

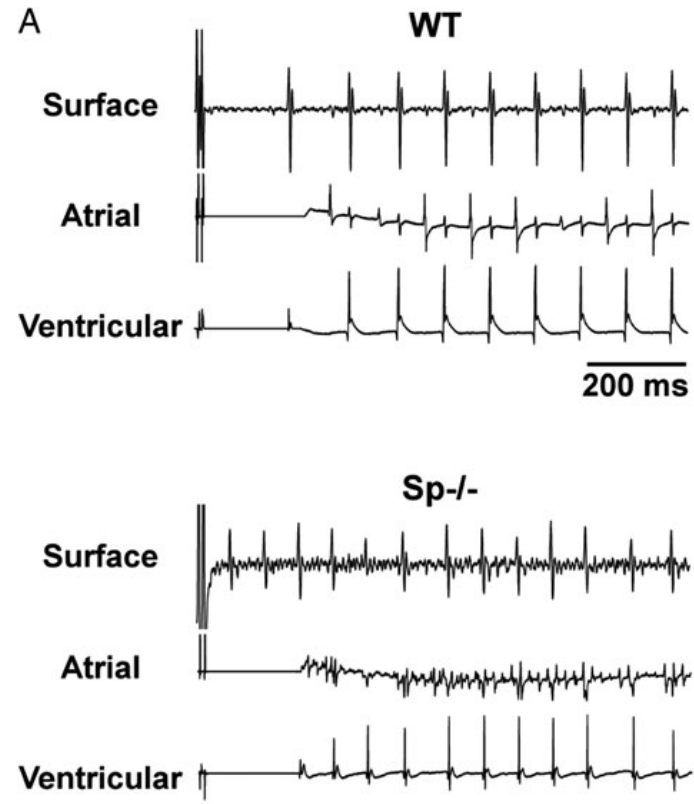
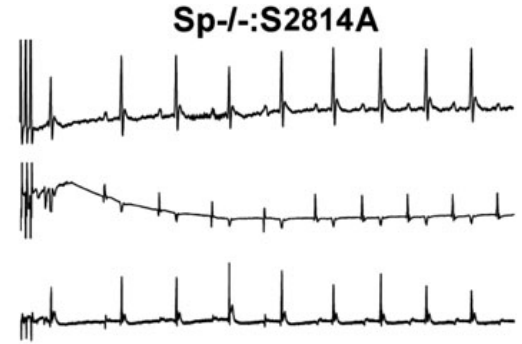

B

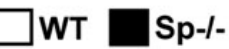

$\square$ Sp-I-:S2814A

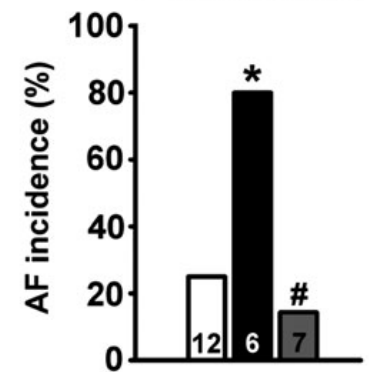

Figure 6 Impaired regulation of RyR2 by PP1 increased AF susceptibility in mice. (A) Representative simultaneous recordings of surface ECG and intracardiac electrograms (atrial and ventricular) after burst pacing. (B) Bar graph summarizing the incidence of pacing-induced $\mathrm{AF}$ in $\mathrm{WT}$, Sp ${ }^{-1-}$, and $\mathrm{Sp}^{-1-}$ :S2814A mice. Numbers in the bars indicate the number of mice studied. ${ }^{*} P<0.05 \mathrm{vs}$. WT. ${ }^{\#} P<0.05 \mathrm{vs}$. Sp ${ }^{-1-}$.
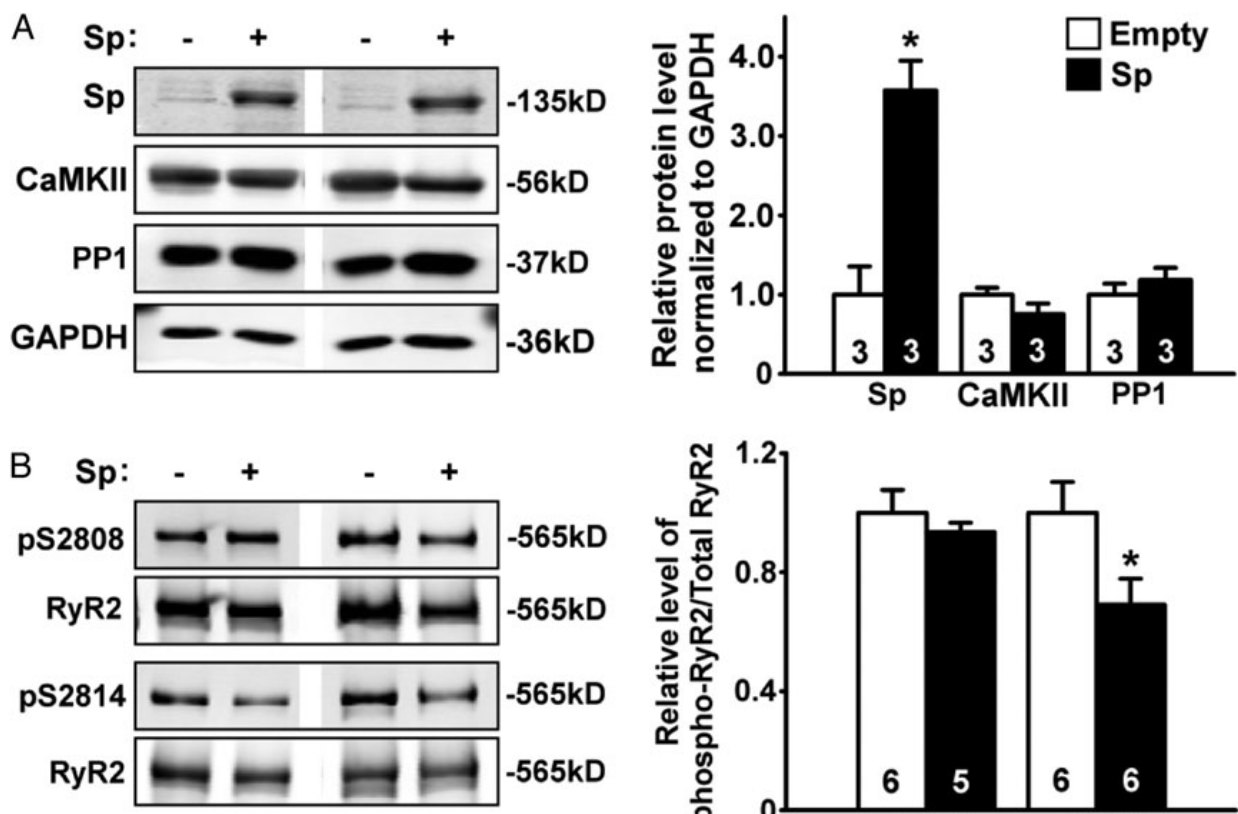

$-565 k D$

$-565 k D$

$-565 k D$

$-565 k D$

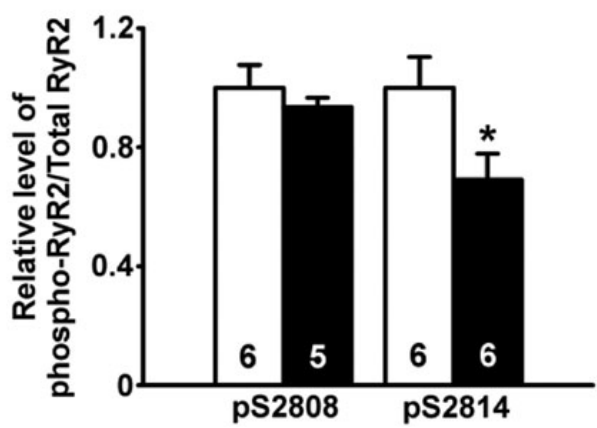

Figure 7 Overexpression of spinophilin decreases RyR2 phosphorylation at S2814. (A) Representative western blots and summary bar graphs showing overexpression of spinophilin in HEK293 cells co-transfected with spinophilin (Sp), RyR2, and CaMKII. Empty, empty pcDNA3.1 vector. (B) Representative western blots and summary bar graphs showing a significant decrease in RyR2 phosphorylation at S2814 but not at S2808 in HEK293 cells co-transfected with spinophilin, RyR2, and CaMKII. Numbers in the bars indicate the number of independent co-transfection experiments. $* P<0.05$ vs. WT.

spinophilin overexpression (Figure 7B). Taken together with our previous findings, this further supports the notion that spinophilin targets PP1 to dephosphorylate RyR2 specifically at S2814 and not at S2808.

\section{Discussion}

Previous studies have described the importance of enhanced RyR2dependent $\mathrm{SR} \mathrm{Ca}^{2+}$ release in the pathogenesis of $\mathrm{AF}^{24,25}$ However, 
the molecular mechanisms underlying enhanced RyR2 activity have remained incompletely understood and quite controversial. ${ }^{1,6,7,10}$ Here, we demonstrated for the first time an important role for the local regulation of PP1 in modulating RyR2 activity in the atria. Our data revealed that when this local regulation is disrupted, RyR2 becomes hyperphosphorylated at S2814 and also hyperactive leading to enhanced $\mathrm{SR} \mathrm{Ca}^{2+}$ release in atrial myocytes. Furthermore, reduced PP1 binding to RyR2 also increased both atrial ectopic events and the susceptibility to pacing-induced AF. Taken together, these data support the novel concept that reduced PP1-dependent dephosphorylation of RyR2 can promote triggered activity and enhance AF susceptibility in mice.

\subsection{Abnormal RyR2 function in AF}

The mechanisms underlying AF are complex, involving both spontaneous ectopic firing and impulse re-entry through the atria. ${ }^{26}$ Abnormal $\mathrm{SR} \mathrm{Ca}{ }^{2+}$ release is now believed to be a main cause of atrial ectopy, and sustained ectopic activity may create a rapid local 'driver' that promotes fibrillatory conduction and AF. ${ }^{26,27}$ Delayed afterdepolarizations, which result from enhanced diastolic SR $\mathrm{Ca}^{2+}$ leak, constitute the most important cause of focal atrial activity. Increased SR Ca ${ }^{2+}$ leak could theoretically result from RyR2 dysfunction and/or SR $\mathrm{Ca}^{2+}$ overload, although there is currently no experimental evidence to support the SR overload hypothesis. ${ }^{8,9,28}$ On the other hand, biochemical analysis of atrial samples from AF patients revealed profound remodelling of the RyR2 macromolecular complex. ${ }^{26}$ For example, the level of FKBP12.6 bound to RyR2 was decreased in AF patients, which might contribute to enhanced RyR2 activity. ${ }^{7}$ Moreover, RyR2 phosphorylation at S2808 (mainly due to PKA) and S2814 (mainly due to CaMKII) was increased in chronic AF patients. ${ }^{6,7,9}$ Furthermore, pharmacological inhibition of CaMKII but not PKA suppressed aberrant diastolic SR $\mathrm{Ca}^{2+}$ release events in atrial myocytes isolated from AF patients. This suggests that enhanced CaMKII activity and S2814 phosphorylation are causally linked to $\mathrm{Ca}^{2+}$ release defect. ${ }^{9}$ Furthermore, genetic inhibition of S2814 phosphorylation on RyR2 suppressed atrial ectopy and AF induction in S2814A knockin mice, as also is evident in our present study (Figures 5 and 6). 6,18 Taken together, these studies support the model that enhanced phosphorylation of RyR2 at S2814 promotes AF.

\subsection{Emerging role of PPs in AF}

The potential role of differential regulation of RyR2 by PPs has not been well studied, in particular in the context of AF. Klein et al. ${ }^{29}$ demonstrated an enhanced $\mathrm{L}$-type $\mathrm{Ca}^{2+}$ current $\left(\mathrm{LTCC}, \mathrm{I}_{\mathrm{Ca}, \mathrm{L}}\right)$ in atrial myocytes from $\mathrm{AF}$ patients. The PP1/PP2A inhibitor okadaic acid (OA) was able to increase LTCC Po only in control but not in AF patients, suggesting an impaired PP-mediated regulation of LTCC. In contrast, Christ et al. ${ }^{30}$ demonstrated that reduced LTCC current in AF patients was caused by increased PP activity, since $O A$ increased basal $I_{C a, L}$ more effectively in $A F$ than in control patients. Whereas mRNA levels of PP1 and PP2A isoforms were decreased, PP1 protein levels were unaltered while PP2 protein levels were increased in AF patients. ${ }^{30} \mathrm{El}$-Armouche et al. ${ }^{1}$ also found a higher total activity of both PP1 and PP2A in patients with chronic AF, but reported inhomogeneous changes of protein phosphorylation levels in different subcellular compartments. For example, PKA phosphorylation of myosin-binding protein-C at S282 was reduced, troponin I phosphorylation was preserved, and phosphorylation of PLN at S16 and T17 was enhanced. ${ }^{1}$ Furthermore, there was a 10 -fold increase in the ratio of T35-phosphorylated to total PP1 inhibitor I-1, implying that PP1 is inhibited within the SR, consistent with hyperphosphorylated PLN and RyR2. ${ }^{1}$ Thus, these prior studies indicate substantial inhomogeneities in PP protein and activity levels across subcellular compartments.

To specifically study the effects of PP1 regulation on RyR2 in the context of AF, we used a model with altered PP1 binding to RyR2 by genetic ablation of the PP1-regulatory subunit spinophilin. ${ }^{11,14}$ This approach enabled us to isolate the effect of PP1 catalytic activity on RyR2, since spinophilin only targets PP1c to RyR2, whereas another PP1-regulatory subunit (PPP1R3A, Rgl) targets PP1c to PLN. ${ }^{11,31}$ Consistent with this model, we showed that, in the absence of spinophilin, RyR2 was hyperphosphorylated while the phosphorylation of PLN, another SR protein, was unchanged (Figure 2A and see Supplementary material online, Figure S3). In addition, our data demonstrated that spinophilin-PP1 primarily dephosphorylates S2814 but not S2808 on RyR2, since only S2814 phosphorylation was enhanced in $\mathrm{Sp}^{-1-}$ mice (Figure 2A). To further validate this model, we showed that overexpression of spinophilin in HEK293 cells led to a selective decrease in the phosphorylation of RyR2 at S2814 but not at S2808 (Figure 7). Taken together, our study is the first in vivo and functional demonstration that spinophilin serves as the PP1-regulatory subunit targeting PP1c to RyR2. Similar findings were originally reported in a recombinant expression system using fusion proteins and mutagenesis. ${ }^{11}$

At first, we were surprised by the finding that RyR2 became hyperphosphorylated at S2814 and not at S2808 because of the current understanding in the field that PP1 primarily regulates S2808 and PP2A primarily dephosphorylates S2814. This understanding is illustrated by one previous study in which immunoprecipitated RyR2 was incubated with either PP1 or PP2A in vitro, and rat myocytes that were exposed to the general PP inhibitor OA. ${ }^{21}$ The authors concluded that while PP1 appeared to more efficiently dephosphorylate $\$ 2808$ and PP2A S2814, both PPs could completely dephosphorylate both sites. $^{21}$ To further complicate the matter, a few other studies have investigated the effect of PP on RyR2 activity with conflicting results. duBell et al. ${ }^{32}$ applied PP1 and PP2A catalytic subunits to patched myocytes and suggested that PP1 and PP2A could decrease excitation-contraction coupling gain by dephosphorylating RyR2. Similarly, Sonnleitner et al. ${ }^{33}$ showed using a lipid bilayer system that PP1 treated RyR1 (isolated from rabbit skeletal muscles) has decreased single-channel activity. However, in contrast to this understanding, Terentyev et al. ${ }^{34}$ reported that PP1 actually increased diastolic RyR2 activity using myocytes permeabilized with saponin and a lipid bilayer system. Finally, Carter et al. ${ }^{35}$ reported the paradoxical finding that both PKA phosphorylation and PP1 dephosphorylation of RyR2 increase its activity in a lipid bilayer, suggesting a non-linear relationship between phosphorylation state and activity.

In light of these previous studies, all of which manipulated the PP1 catalytic subunit by either applying it exogenously or inhibiting it globally with OA, the present study specifically disrupts PP1's binding to RyR2 in the native cardiac environment without altering the total PP1c level or activity. In this system, we found that RyR2 S2814 is preferentially dephosphorylated by PP1, and that this increases RyR2 activity with functional consequences both at the myocyte and at the whole animal level. In other words, our data suggest that, in the native cardiac environment, PP1 mainly regulates RyR2 S2814 and not S2808 in vivo. On the other hand, PP2A may also target S2814 on RyR2 as suggested by a recent study that targeted a PP2A regulatory subunit B56 $\alpha$ via miR-1, although that study was limited to isolated myocytes and not in the whole animal. ${ }^{36}$ 


\subsection{Potential alternative targeting mechanism of PP1 to RyR2}

Our data revealed some residual binding of PP1 to RyR2 in the Sp ${ }^{-1-}$ mice (Figure 1C), suggesting that there might be an alternative mechanism by which PP1 interacts with RyR2. Targeting of PP1 to RyR2 via spinophilin was first demonstrated by Marx et al. ${ }^{11}$ using in vitro assays. However, an earlier study by Zhao et al. ${ }^{37}$ using affinity columns showed that PP1 can bind directly to both RyR1 and RyR2. Thus, it is possible that some PP1 might bind directly to RyR2. Alternatively, it is possible that additional regulatory subunits may also target PP1 to the RyR2 macromolecular complex, since PP1c binds a number of other regulatory subunits in the heart (unpublished data). Further experimental studies are needed to explore such possibilities.

\subsection{Potential limitations}

We took advantage of the $\mathrm{Sp}^{-1-}$ mice to study PP1's local regulation of RyR2. However, the gene ablation in this mouse model was not atrialspecific. ${ }^{14}$ Nevertheless, prior papers and our current results demonstrated no gross abnormalities in the $\mathrm{Sp}^{-1-}$ mice, except for a slightly reduced body weight, heart, and brain size (see Supplementary material online, Figures S5 and S6). ${ }^{14}$ Also, cardiac contractility and ventricular functions were largely unaltered (see Supplementary material online, Table S2). Therefore, atrial arrhythmogenesis is probably not secondary to atrial or ventricular remodelling or extra-cardiac effects. Another potential limitation is that $\mathrm{Sp}^{-1-}$ mice did not exhibit spontaneous AF. Our studies were conducted in 2- to 4-month-old mice, so it is possible that older $\mathrm{Sp}^{-1-}$ mice will be more likely to develop spontaneous AF. On the other hand, it is well recognized that only few transgenic mouse models exhibit spontaneous $\mathrm{AF}^{38}$ Nevertheless, many other genetic mouse models with an enhanced susceptibility to pacing-induced AF have provided valuable information on the molecular pathways involved in atrial ectopy and remodelling. Finally, the residual PP1 bound to RyR2 in the absence of spinophilin may complicate the interpretation of our findings (Figure 1C). Nevertheless, even though this binding between PP1 and RyR2 is not completely abolished in $\mathrm{Sp}^{-1-}$ mice, it is still significantly decreased or 'impaired' and thereby supports the overall conclusion of this study.

\subsection{Conclusion}

The results of this study clearly demonstrate for the first time that the local regulation by PP1 is required for the homeostasis of RyR2 phosphorylation in vivo in mouse atria. Specific dissociation of PP1c from the RyR2 macromolecular complex promotes hyperphosphorylation of RyR2, which is associated with enhanced RyR2 activity, spontaneous $\mathrm{Ca}^{2+}$ release events, atrial ectopy, and enhanced AF susceptibility in mice. These phenotypes both at the cellular and organ levels can be rescued pharmacologically by CaMKII inhibition or genetically by ablation of the RyR2 S2814 phosphorylation site. Taken together, our data suggest that (i) PP1 directly opposes CaMKIl's action on RyR2 S2814 in vivo and (ii) disruption of PP1's regulation on RyR2 contributes to AF susceptibility. This represents a novel understanding of AF pathogenesis and offers insights into new therapeutic strategies such as strengthening the targeting of PP1 to RyR2.

\section{Supplementary material}

Supplementary material is available at Cardiovascular Research online.

\section{Acknowledgements}

We thank Drs Marc Flajolet and Paul Greengard (Rockefeller University) for providing the $\mathrm{Sp}^{-1-}$ mice, Dr Andrew Marks (Columbia University) for the pCMV5-hRyR2 vector, Dr Mark Anderson (University of lowa) for the $\mathrm{PSR} \alpha-\mathrm{CaMKII \delta c} \mathrm{vector,} \mathrm{and} \mathrm{the} \mathrm{Mouse} \mathrm{Phenotyping} \mathrm{Core} \mathrm{at} \mathrm{the}$ Baylor College of Medicine for help with the echocardiography studies.

Conflict of interest: none declared.

\section{Funding}

This work was supported by the American Heart Association (12PRE11700012 to D.Y.C., 12BGIA12050207 to N.L., and 13EIA14560061 to X.H.T.W.); the National Institutes of Health (HL089598, HL091947, and HL117641 to X.H.T.W; GM069234 to K.M.A.); the Muscular Dystrophy Association (to X.H.T.W.); the Fondation Leducq ('Alliance for CaMKII Signaling in Heart' to X.H.T.W, 'European North-American Atrial Fibrillation Research Alliance' to D.D.); the Juanita P. Quigley endowed chair in cardiology (to X.H.T.W.); the European Network for Translational Research in Atrial Fibrillation (EUTRAF; 261057 to D.D.); and the Baylor College of Medicine Medical Scientist Training Program Caskey Scholarship (to D.Y.C.).

\section{References}

1. El-Armouche A, Boknik P, Eschenhagen T, Carrier L, Knaut M, Ravens U, Dobrev D Molecular determinants of altered $\mathrm{Ca}^{2+}$ handling in human chronic atrial fibrillation. Circulation 2006;114:670-680.

2. Heroes E, Lesage B, Gornemann J, Beullens M, Van Meervelt L, Bollen M. The PP1 binding code: a molecular-lego strategy that governs specificity. FEBS J 2013;280:584-595.

3. Carr AN, Schmidt AG, Suzuki Y, del Monte F, Sato Y, Lanner C, Breeden K, Jing SL, Allen PB, Greengard P, Yatani A, Hoit BD, Grupp IL, Hajjar RJ, DePaoli-Roach AA, Kranias EG. Type 1 phosphatase, a negative regulator of cardiac function. Mol Cell Biol 2002;22:4124-4135.

4. El-Armouche A, Wittkopper K, Degenhardt F, Weinberger F, Didie M, Melnychenko I, Grimm M, Peeck M, Zimmermann WH, Unsold B, Hasenfuss G, Dobrev D, Eschenhagen T. Phosphatase inhibitor-1-deficient mice are protected from catecholamine-induced arrhythmias and myocardial hypertrophy. Cardiovasc Res 2008; 80:396-406.

5. Kirchhefer U, Baba HA, Boknik P, Breeden KM, Mavila N, Bruchert N, Justus I, Matus M, Schmitz W, Depaoli-Roach AA, Neumann J. Enhanced cardiac function in mice overexpressing protein phosphatase Inhibitor-2. Cardiovasc Res 2005;68:98-108.

6. Chelu MG, Sarma S, Sood S, Wang S, van Oort RJ, Skapura DG, Li N, Santonastasi M, Muller FU, Schmitz W, Schotten U, Anderson ME, Valderrabano M, Dobrev D, Wehrens $\mathrm{XH}$. Calmodulin kinase II-mediated sarcoplasmic reticulum $\mathrm{Ca}^{2+}$ leak promotes atrial fibrillation in mice. J Clin Invest 2009;119:1940-1951.

7. Vest JA, Wehrens XH, Reiken SR, Lehnart SE, Dobrev D, Chandra P, Danilo P, Ravens U, Rosen MR, Marks AR. Defective cardiac ryanodine receptor regulation during atrial fibrillation. Circulation 2005;111:2025-2032.

8. Hove-Madsen L, Llach A, Bayes-Genis A, Roura S, Rodriguez Font E, Aris A, Cinca J. Atrial fibrillation is associated with increased spontaneous calcium release from the sarcoplasmic reticulum in human atrial myocytes. Circulation 2004;110:1358-1363.

9. Voigt N, Li N, Wang Q, Wang W, Trafford AW, Abu-Taha I, Sun Q, Wieland T, Ravens U, Nattel S, Wehrens XH, Dobrev D. Enhanced sarcoplasmic reticulum $\mathrm{Ca}^{2+}$ leak and increased $\mathrm{Na}^{+}-\mathrm{Ca}^{2+}$ exchanger function underlie delayed afterdepolarizations in patients with chronic atrial fibrillation. Circulation 2012;125:2059-2070.

10. Marx SO, Reiken S, Hisamatsu Y, Jayaraman T, Burkhoff D, Rosemblit N, Marks AR. PKA phosphorylation dissociates FKBP12.6 from the calcium release channel (ryanodine receptor): defective regulation in failing hearts. Cell 2000;101:365-376.

11. Marx SO, Reiken S, Hisamatsu Y, Gaburjakova M, Gaburjakova J, Yang YM, Rosemblit N, Marks AR. Phosphorylation-dependent regulation of ryanodine receptors: a novel role for leucine/isoleucine zippers. J Cell Biol 2001;153:699-708.

12. El-Armouche A, Pamminger T, Ditz D, Zolk O, Eschenhagen T. Decreased protein and phosphorylation level of the protein phosphatase inhibitor-1 in failing human hearts. Cardiovasc Res 2004;61:87-93.

13. Gupta RC, Mishra S, Rastogi S, Imai M, Habib O, Sabbah HN. Cardiac SR-coupled PP1 activity and expression are increased and inhibitor 1 protein expression is decreased in failing hearts. Am J Physiol Heart Circ Physiol 2003;285:H2373-H2381.

14. Feng J, Yan Z, Ferreira A, Tomizawa K, Liauw JA, Zhuo M, Allen PB, Ouimet CC, Greengard P. Spinophilin regulates the formation and function of dendritic spines. Proc Natl Acad Sci USA 2000;97:9287-9292.

15. Reynolds JO, Chiang DY, Wang W, Beavers DL, Dixit SS, Skapura DG, Landstrom AP Song LS, Ackerman MJ, Wehrens $\mathrm{XH}$. Junctophilin-2 is necessary for T-tubule maturation during mouse heart development. Cardiovasc Res 2013;100:44-53. 
16. Zhou Q, Wang QL, Meng X, Shu Y, jiang T, Wagenknecht T, Yin CC, Sui SF, Liu Z. Structural and functional characterization of ryanodine receptor-natrin toxin interaction. Biophys J 2008;95:4289-4299.

17. Wehrens XH, Lehnart SE, Huang F, Vest JA, Reiken SR, Mohler PJ, Sun J, Guatimosim S, Song LS, Rosemblit N, D’Armiento JM, Napolitano C, Memmi M, Priori SG, Lederer WJ, Marks AR. FKBP12.6 deficiency and defective calcium release channel (ryanodine receptor) function linked to exercise-induced sudden cardiac death. Cell 2003;113: $829-840$.

18. Li N, Wang T, Wang W, Cutler MJ, Wang Q, Voigt N, Rosenbaum DS, Dobrev D, Wehrens $\mathrm{XH}$. Inhibition of CaMKII phosphorylation of RyR2 prevents induction of atrial fibrillation in FKBP12.6 knockout mice. Circ Res 2012;110:465-470.

19. McCauley MD, Wehrens XH. Ambulatory ECG recording in mice. J Vis Exp 2010;39: 1729.

20. Li N, Wehrens $X H$. Programmed electrical stimulation in mice. J Vis Exp 2010;39: 1730.

21. Huke S, Bers DM. Ryanodine receptor phosphorylation at Serine 2030,2808 and 2814 in rat cardiomyocytes. Biochem Biophys Res Commun 2008;376:80-85.

22. Picht E, Zima AV, Blatter LA, Bers DM. SparkMaster: automated calcium spark analysis with ImageJ. Am J Physiol Cell Physiol 2007;293:C1073-C1081.

23. Sood S, Chelu MG, van Oort RJ, Skapura D, Santonastasi M, Dobrev D, Wehrens XH. Intracellular calcium leak due to FKBP12.6 deficiency in mice facilitates the inducibility of atrial fibrillation. Heart Rhythm 2008;5:1047-1054.

24. Wehrens $\mathrm{XH}$, Ather $\mathrm{S}$, Dobrev D. Role of abnormal sarcoplasmic reticulum function in atrial fibrillation. Therapy 2010;7:147-158.

25. Li N, Chiang DY, Wang S, Wang Q, Sun L, Voigt N, Respress JL, Ather S, Skapura DG, Jordan VK, Horrigan FT, Schmitz W, Muller FU, Valderrabano M, Nattel S, Dobrev D, Wehrens $\mathrm{XH}$. Ryanodine receptor-mediated calcium leak drives progressive development of an atrial fibrillation substrate in a transgenic mouse model. Circulation 2014; 129:1276-1285

26. Wakili R, Voigt N, Kaab S, Dobrev D, Nattel S. Recent advances in the molecular pathophysiology of atrial fibrillation. J Clin Invest 2011;121:2955-2968.

27. Dobrev D, Nattel S. New antiarrhythmic drugs for treatment of atrial fibrillation. Lancet 2010;375:1212-1223.

28. Neef S, Dybkova N, Sossalla S, Ort KR, Fluschnik N, Neumann K, Seipelt R, Schondube FA, Hasenfuss G, Maier LS. CaMKII-dependent diastolic SR $\mathrm{Ca}^{2+}$ leak and elevated diastolic $\mathrm{Ca}^{2+}$ levels in right atrial myocardium of patients with atrial fibrillation. Circ Res 2010;106:1134-1144.
29. Klein G, Schroder F, Vogler D, Schaefer A, Haverich A, Schieffer B, Korte T, Drexler H. Increased open probability of single cardiac L-type calcium channels in patients with chronic atrial fibrillation. Role of phosphatase 2A. Cardiovasc Res 2003;59:37-45.

30. Christ T, Boknik P, Wohrl S, Wettwer E, Graf EM, Bosch RF, Knaut M, Schmitz W, Ravens $U$, Dobrev D. L-type $\mathrm{Ca}^{2+}$ current downregulation in chronic human atrial fibrillation is associated with increased activity of protein phosphatases. Circulation 2004; 110:2651-2657.

31. Berrebi-Bertrand I, Souchet M, Camelin JC, Laville MP, Calmels T, Bril A. Biophysical interaction between phospholamban and protein phosphatase 1 regulatory subunit GM. FEBS Lett 1998;439:224-230.

32. duBell WH, Lederer WJ, Rogers TB. Dynamic modulation of excitation-contraction coupling by protein phosphatases in rat ventricular myocytes. J Physiol 1996;493(Pt 3): $793-800$.

33. Sonnleitner A, Fleischer S, Schindler H. Gating of the skeletal calcium release channel by ATP is inhibited by protein phosphatase 1 but not by $\mathrm{Mg}^{2+}$. Cell Calcium 1997;21: $283-290$.

34. Terentyev D, Viatchenko-Karpinski S, Gyorke I, Terentyeva R, Gyorke S. Protein phosphatases decrease sarcoplasmic reticulum calcium content by stimulating calcium release in cardiac myocytes. J Physiol 2003;552:109-118.

35. Carter S, Colyer J, Sitsapesan R. Maximum phosphorylation of the cardiac ryanodine receptor at serine- 2809 by protein kinase a produces unique modifications to channel gating and conductance not observed at lower levels of phosphorylation. Circ Res 2006;98:1506-1513.

36. Terentyev D, Belevych AE, Terentyeva R, Martin MM, Malana GE, Kuhn DE, AbdellatifM, Feldman DS, Elton TS, Gyorke S. miR-1 overexpression enhances $\mathrm{Ca}(2+)$ release and promotes cardiac arrhythmogenesis by targeting PP2A regulatory subunit B56alpha and causing CaMKII-dependent hyperphosphorylation of RyR2. Circ Res 2009;104: $514-521$.

37. Zhao S, Brandt NR, Caswell AH, Lee EY. Binding of the catalytic subunit of protein phosphatase- 1 to the ryanodine-sensitive calcium release channel protein. Biochemistry 1998;37:18102-18109.

38. Kirchhof P, Marijon E, Fabritz L, Li N, Wang W, Wang T, Schulte K, Hanstein J, Schulte JS, Vogel M, Mougenot N, Laakmann S, Fortmueller L, Eckstein J, Verheule S, Kaese S, Staab A, Grote-Wessels S, Schotten U, Moubarak G, Wehrens XH, Schmitz W, Hatem S, Muller FU. Overexpression of cAMP-response element modulator causes abnormal growth and development of the atrial myocardium resulting in a substrate for sustained atrial fibrillation in mice. Int / Cardiol 2011;166:366-374. 